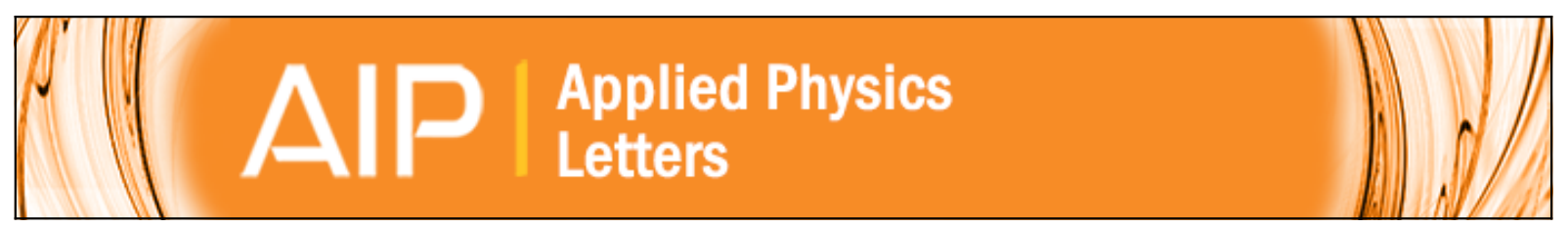

Tunable stiffness of electrorheological elastomers by designing mesostructures

Changyong Cao and Xuanhe Zhao

Citation: Applied Physics Letters 103, 041901 (2013); doi: 10.1063/1.4816287

View online: $\mathrm{http} / / / \mathrm{dx}$.doi.org/10.1063/1.4816287

View Table of Contents: http://scitation.aip.org/content/aip/journal/apl/103/4?ver=pdfcov

Published by the AIP Publishing

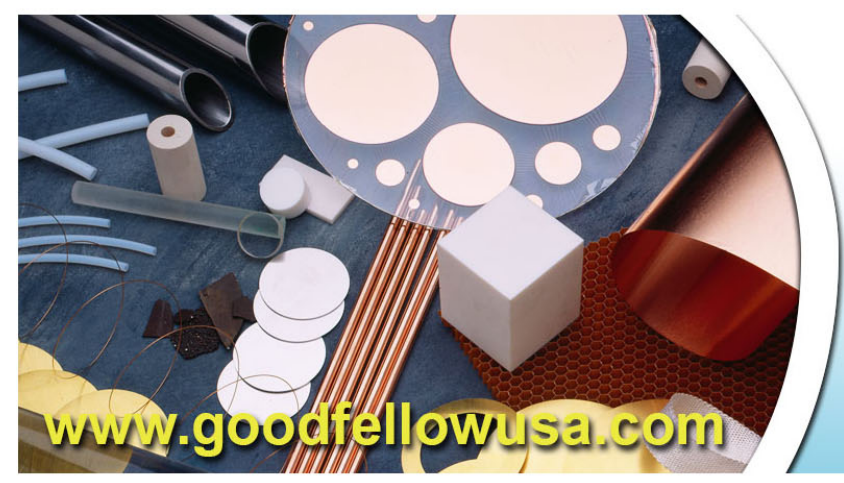

BoOdFEl/OW metals • ceramics $\cdot$ polymers composites $\bullet$ compounds • glasses

Save $5 \%$ Buy online 70,000 products $\cdot$ Fast shipping 


\title{
Tunable stiffness of electrorheological elastomers by designing mesostructures
}

\author{
Changyong $\mathrm{Cao}^{1,2}$ and Xuanhe Zhao ${ }^{1, \mathrm{a})}$ \\ ${ }^{1}$ Soft Active Materials Laboratory, Department of Mechanical Engineering and Materials Science, \\ Duke University, Durham, North Carolina 27708, USA \\ ${ }^{2}$ Research School of Engineering, Australian National University, Canberra, ACT 0200, Australia
}

(Received 30 May 2013; accepted 5 July 2013; published online 22 July 2013)

\begin{abstract}
Electrorheological elastomers have broad and important applications. While existing studies mostly focus on microstructures of electrorheological elastomers, their mesoscale structures have been rarely investigated. We present a theory on the design of mesostructures of electrorheological elastomers that consist of two phases with different permittivity. We show that the deformation of elastomers can reorient their mesostructures, which consequently results in variations of their effective permittivity, leading to stiffening, softening, or instability of the elastomer. Optimal design of the mesostructures can give giant tunable stiffness. Our theoretical model is further validated by results from numerical simulations. (C) 2013 AIP Publishing LLC .
\end{abstract}

[http://dx.doi.org/10.1063/1.4816287]

Electrorheological elastomers (EREs) and magnetorheological elastomers (MREs), which can vary their moduli under applied electric and magnetic fields, have diverse applications ranging from actuators and energy harvesters to controllable dampers. ${ }^{1-5}$ Existing ERE and MRE are mostly fabricated by dispersing micrometer-size filler particles with high electrical permittivity or magnetic permeability in elastomer matrices. ${ }^{1-4,6}$ Since interactions of the filler particles under electric or magnetic fields induce stiffening or softening of the elastomers, microstructures of ERE and MRE play an important role in their tunable stiffness. ${ }^{7-10}$ For example, stronger magnetorheological or electrorheological effects have been observed in MRE or ERE, where the filler particles are aligned along one direction. ${ }^{8-10}$ Various theoretical and numerical models have been developed to account for the effects of ERE and MRE's microstructures. ${ }^{11-19}$ Despite the intensive research, it is still technically and economically challenging to precisely control the microstructures (e.g. exact locations of filler particles) of ERE and MRE.

On the other hand, the mesoscale structures of ERE and MRE can be easily controlled with existing fabrication techniques. For instance, polymers that consist of layers with well-defined thickness (e.g., $10 \mu \mathrm{m} \sim 1 \mathrm{~mm})$ and permittivity have been fabricated over large areas. ${ }^{20-22}$ Such mesostructures have demonstrated interesting phenomena such as instabilities ${ }^{23}$ and patterning, ${ }^{24}$ when subjected to applied electric fields. Nevertheless, the effects of ERE and MRE's mesostructures on their electrorheological or magnetorheological behaviors have not been investigated.

This paper is focused on the design of mesostructures of ERE. Using theory and numerical simulation, we demonstrate that giant tunable stiffness can be achieved by designing the mesostructures of ERE. The essential idea of the design is illustrated in Fig. 1. An electric field is applied on an ERE with anisotropic mesostructures. The applied field has an angle with the orientation of the mesostructure at its

\footnotetext{
a)Email: xz69@duke.edu
}

undeformed state. Deformation of the elastomer varies the angle between the applied field and the mesostructure. This angle variation changes the electrostatic potential energy of the elastomer, leading to stiffening, softening, or instability of the elastomer.

Let us consider a layer of an ERE with thickness $H$ and surface area $A$ as illustrated in Fig. 2(a). The top and bottom surfaces of the elastomer are bonded to two rigid conductive plates. An electrical voltage $\Phi$ is applied between the plates by a battery, and a horizontal force $F$ is applied on the top plate. Due to the constraint of the rigid plates, the elastomer undergoes simple shear deformation. The voltage and the force induce a magnitude of electric charge $Q$ on each plate and a shear strain $\Delta \theta$ in the elastomer (Fig. 2(a)). The elastomer is taken to be incompressible, so that its thickness does not change during the deformation. The applied force, the battery, and the elastomer constitute a thermodynamic system. The free energy of the system per unit volume of the elastomer can be expressed as ${ }^{25}$

$$
\Pi=\frac{1}{2} \bar{G} \Delta \theta^{2}+\frac{\bar{D}^{2}}{2 \varepsilon f(\Delta \theta, \mathbf{X})}-\tau \Delta \theta-\bar{E} \bar{D}
$$

where $\bar{G}$ is the average shear modulus of the elastomer, $\tau$ $=F / A$ the applied shear stress, $\bar{E}=\Phi / H$ the average

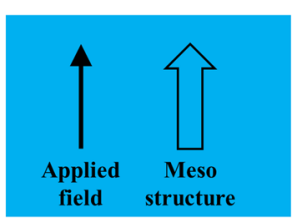

Undeformed State

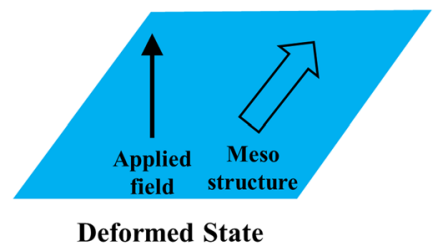

FIG. 1. Schematic illustrations of an ERE with anisotropic mesotructures. The applied electric field has an angle with the orientation of the mesostructure at its undeformed state. Deformation of the elastomer varies the angle between the applied field and the mesostructure, changing the electrostatic potential energy of the elastomer. 
(a)

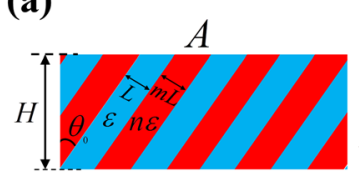

(c)

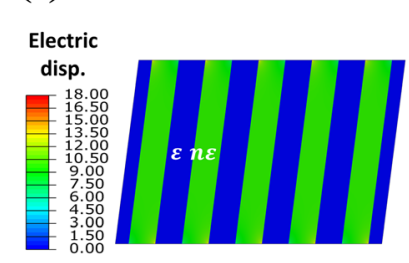

(b)

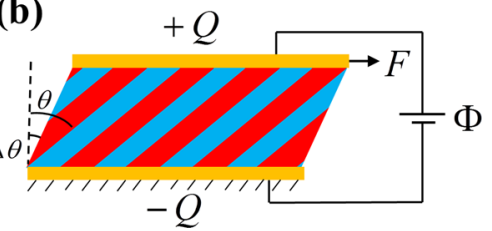

(d)

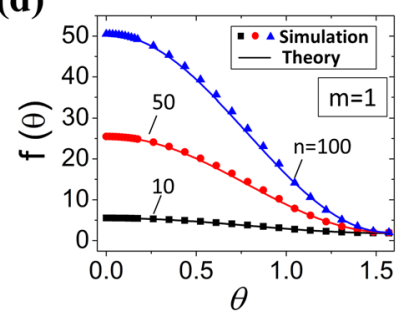

FIG. 2. An ERE with layered mesostructures at undeformed state (a) and undergoing simple shear deformation by an applied force and a voltage (b). The distribution of electric displacement in the layered mesostructure calculated by a finite-element model (c). The normalized effective permittivity $f$ as functions of layer orientation $\theta$ calculated by the numerical model and the theory (d).

electric field, $\bar{D}=Q / A$ the average electric displacement, $\varepsilon$ a typical scale of the permittivity of the elastomer (e.g., the permittivity of one phase), $f(\Delta \theta, \mathbf{X})$ a non-dimensional function, and $\mathbf{X}$ represents a set of coefficients that characterize the mesostructure of the elastomer. It is noted that, to illustrate the essential design ideas, here we assume the elastomer undergoes small shear deformation.

The thermodynamic system has two variables, $\Delta \theta$ and $\bar{D}$, and two control parameters, $\tau$ and $\bar{E}$. At equilibrium, thermodynamics dictates $\partial \Pi / \partial \bar{D}=0$ and $\partial \Pi / \partial \Delta \theta=0$, which, respectively, gives

$$
\begin{gathered}
\bar{E}=\frac{\bar{D}}{\varepsilon f}, \\
\tau=\bar{G} \Delta \theta-\frac{\bar{D}^{2}}{2 \varepsilon} \frac{f^{\prime}}{f^{2}},
\end{gathered}
$$

where $f^{\prime}=\partial f / \partial \Delta \theta$. From Eq. (2), it is evident that $\varepsilon f$ gives the effective permittivity of the elastomer, i.e., $\bar{\varepsilon}=\varepsilon f$ $=\bar{D} / \bar{E}$. The use of effective permittivity $\bar{\varepsilon}$ in Eqs. (1) and (2) will be validated by numerical models that can be used to directly calculate $\Phi$ and $Q$ so as to evaluate $\bar{E}$ and $\bar{D}$, respectively (Figs. 2(c) and 2(d)). By substituting Eq. (2) into Eq. (3), we obtain the relation between the shear stress and shear strain of the elastomer under an applied voltage,

$$
\tau=\bar{G} \Delta \theta-\frac{\varepsilon \bar{E}^{2}}{2} f^{\prime} .
$$

Therefore, the effective modulus of the elastomer under an applied voltage can be calculated as

$$
G_{e f f}=\left.\frac{\partial \tau}{\partial \Delta \theta}\right|_{\bar{E}}=\bar{G}+\frac{\varepsilon \bar{E}^{2}}{2} K_{E R}
$$

where $K_{E R}=-f^{\prime \prime}=-\partial^{2} f / \partial \Delta \theta^{2}$ is defined as the electrorheological coefficient. In addition, the stability of the thermodynamic system requires its Hessian matrix, ${ }^{25,26}$

$$
\mathbf{H}=\left[\begin{array}{cc}
\frac{\partial \Pi^{2}}{\partial \Delta \theta^{2}} & \frac{\partial \Pi^{2}}{\partial \Delta \theta \partial \bar{D}} \\
\frac{\partial \Pi^{2}}{\partial \Delta \theta \partial \bar{D}} & \frac{\partial \Pi^{2}}{\partial \bar{D}^{2}}
\end{array}\right]
$$

to be positive definite.

From Eq. (5), we know that a higher electrorheological coefficient $K_{E R}$ (i.e., $-f^{\prime \prime}$ ) will lead to higher tunable stiffness under an applied voltage, given that the system satisfies stability condition. The effective permittivity and electrostatic energy of isotropic dielectrics are independent of their orientations, and therefore isotropic dielectrics have $K_{E R}=0$. In order to achieve high values of $K_{E R}$, we need to design anisotropic mesostructures of elastomer to make its effective permittivity highly dependent on orientation.

It is well known that layers with different dielectrics arranged in serial and parallel configures give the lower and upper limits of the composite's effective permittivity. ${ }^{27}$ Therefore, in the current study, we choose a layered mesostructure of two dielectrics to constitute the elastomer (Fig. 2(a)). As the elastomer is undergoing deformation, the orientation of the layers will vary, potentially leading to high values of $K_{E R}$ and tunable stiffness (Fig. 2(b)).

Let us denote the permittivity of the two phases in the elastomer to be $\varepsilon$ and $n \varepsilon$ and their thicknesses to be $L$ and $m L$, respectively (Fig. 2(a)). The angle between the layers and the vertical direction is $\theta_{0}$ at the undeformed state (Fig. 2(a)) and $\theta$ at the deformed state (Fig. 2(b)). The thickness of the elastomer does not change due to incompressibility, so that the angles $\theta_{0}, \theta$, and $\Delta \theta$ are related via

$$
\tan \theta=\tan \theta_{0}+\tan \Delta \theta
$$

and for small angles of $\theta_{0}$,

$$
\theta \approx \theta_{0}+\Delta \theta
$$

The effective permittivity of the layered structure $\bar{\varepsilon}$ (i.e., $\varepsilon f$ ) can be regarded as a function of variable $\theta$ with coefficients $\varepsilon, n$, and $m$. To calculate the effective permittivity, we construct a numerical model using finite-element software package ABAQUS 6.11. The model calculates the distribution of electric field and electric displacement in layered mesostructures under applied voltage, $\Phi$ (e.g., Fig. 2(c) for $n=100$ and $m=1)$. It can be seen that the electric displacement is localized at layers with high permittivity. An integration of the electric displacement over an electrode gives the induced charge $Q$. Therefore, the effective permittivity of the layered structure can be calculated with the finite-element model. In Fig. 2(d), we give the calculated normalized effective permittivity $f$ as functions of $\theta$ for various values of $n$. As expected, the effective permittivity decreases with $\theta$, such that the parallel and serial configures of layers give the upper and lower limits of $f$, respectively. Considering the symmetry of the function $f(\theta)$, we find it can be relatively accurately approximated by

$$
f(\theta)=\alpha \cos (2 \theta)+\beta,
$$

where $\alpha=[f(0)-f(\pi / 2)] / 2$ and $\beta=[f(0)+f(\pi / 2)] / 2$. The effective permittivity of serial and parallel models of 
dielectric composites gives $f(0)=(1+n m) /(1+m)$ and $f(\pi / 2)=(1+m) /(1+m / n) \cdot{ }^{27}$ From Fig. $2(\mathrm{~d})$, it can be seen that the match between the theory and numerical results is particularly good at small angles of $\theta$.

From Eqs. (5), (7), and (9), we can calculate the electrorheological coefficient as

$$
K_{E R}=4 \alpha \cos (2 \theta)\left(\frac{\partial \theta}{\partial \Delta \theta}\right)^{2}+2 \alpha \sin (2 \theta) \frac{\partial^{2} \theta}{\partial \Delta \theta^{2}}
$$

and for small angles of $\theta_{0}$, considering Eq. (8), we have

$$
K_{E R}=4 \alpha \cos (2 \theta) \text {. }
$$

In Fig. 3(a), we plot the calculated electrorheological coefficient $K_{E R}$ for various values of permittivity ratio according to Eq. (10). Notably, $K_{E R}$ reaches its maximum value at $\theta=0$, giving the highest electrorheological coefficient. The stiffening effect can be qualitatively understood as follow. The parallel configuration of layers (e.g., inset in Fig. 3(b)) gives the lowest electrostatic potential energy of the system. Therefore, to deform the same amount of shear strain, a parallel configuration of layers under applied voltage requires a higher shear stress than the same structure without voltage, meaning voltage-induced stiffening.

Since our goal is to achieve giant tunable stiffness, we focus on the electrorheological coefficient at $\theta=0$, which can be expressed as

$$
K_{E R}(0)=\frac{2 m(n-1)^{2}}{(1+m)(m+n)} .
$$

A simple optimization shows that when $m=\sqrt{n}$, the value of $K_{E R}(0)$ reaches its maximum with respective to $m$, i.e.,

$$
K_{E R}^{\max }(0)=2(\sqrt{n}-1)^{2} .
$$

In Fig. 3(b), we plot the calculated $K_{E R}(0)$ as functions of the permittivity ratio $n$ for various ratios of layer thickness $m$. It can be seen that $K_{E R}(0)$ increases almost linearly with permittivity ratio $n$. Furthermore, for the same $n$, an optimized concentration of the high-permittivity phase (i.e., $m=\sqrt{n}$ ) gives the highest electrorheological coefficient $K_{E R}(0)$.

Dielectrics with high permittivity usually have much higher moduli than elastomer matrices. Deformation of the rigid high-permittivity layers following Fig. 2(b) will lead to (a)

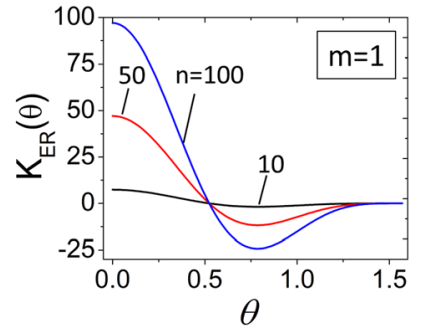

(b)

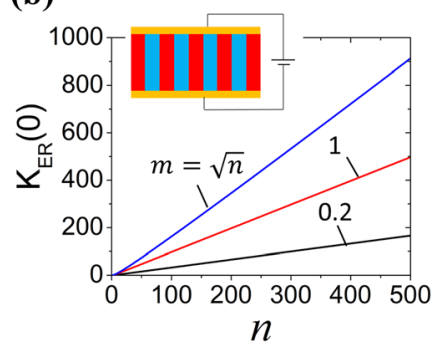

FIG. 3. The calculated electrorheological coefficient $K_{E R}$ as functions of layer orientation $\theta$ (a). At $\theta=0, K_{E R}$ reaches its maximum value. The calculated $K_{E R}(0)$ as functions of $n$ and $m(\mathrm{~b})$. very high effective shear moduli of the mesostructures. To reduce the effective modulus, we propose to vertically embed plates of rigid high-permittivity phases in elastomer matrices as illustrated in Fig. 4(a), which allows rotation of the rigid plates when the mesostructure is under shear deformation. Let us denote the distance from the top/bottom surfaces of the rigid plates to the top/bottom electrodes as $\mathrm{rH}$ (Fig. 4(a)). Using ABAqus 6.11, we develop a model for the plane-strain deformation of the mesostructure to calculate its effective shear modulus $\bar{G}$. Fig. 4(b) shows that when the mesostructure is sheared to rotate the rigid inclusions, only the elastomer adjacent to the electrodes undergoes relatively large deformation. In Fig. 4(c), we give the calculated effective shear modulus $\bar{G}$ for various values of $r$ and $m$. Notably, even for relatively small gaps between inclusions and electrodes and moderate concentration of inclusions, the effective modulus is only a few times higher than the elastomer's modulus (e.g., $\bar{G} / G_{0}=3$ for $r=2 \%$ and $m=0.5$ in Fig. $4(\mathrm{c})$, where $G_{0}$ is the shear modulus of matrix). Therefore, we choose $r=2 \%$ and $m \leq 0.5$ to calculate the electrorheological coefficient $K_{E R}(0)$ of mesostructures with various values of $n$. From Fig. 4(d), it can be seen that $K_{E R}(0)$ still increases monotonically with $n$. Furthermore, a combination of high permittivity and moderate concentration of the inclusions can indeed lead to high values of electrorheological coefficient $\left[e . g ., K_{E R}(0)=150\right.$ for $r=2 \%, m=0.5$, and $n=1000$ in Fig. 4(d)].

Based on the theory and numerical calculation above, we can evaluate the enhancements of shear moduli of various compositions for layered mesostructures. We select the commonly used silicone rubber, polydimethylsiloxane (PDMS), to constitute matrix phase of the mesostructure. Various other polymers or ceramics are chosen as the filler phase of the mesostructure, including VHB (Acrylic Tape from 3M company), natural rubber, fluorogel, Polyvinylidene fluoride (PVDF), ${ }^{28} \mathrm{TiO}_{2}, \mathrm{BaTiO}_{3}$, and lead zirconate titanate (PZT). (a)

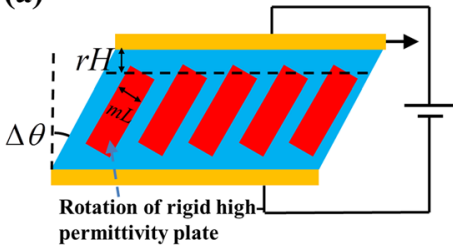

(c)

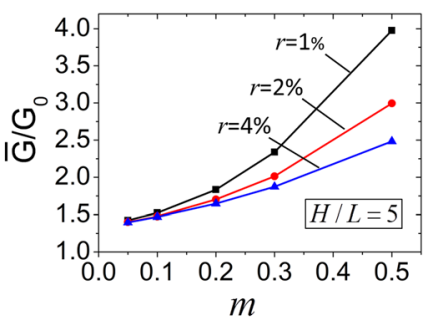

(b)

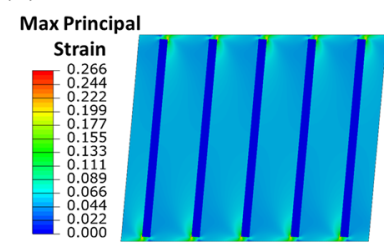

(d)

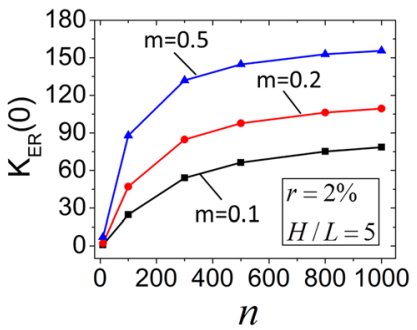

FIG. 4. High-permittivity rigid plates vertically embedded in an elastomer matrix can rotate when the mesostructure is under shear (a). The distribution of principal strain in a mesostructure with $r=2 \%, m=0.5$, and $H / L=5$ under shear strain $\Delta \theta=5^{\circ}$ (b). The calculated effective moduli $\bar{G}$ as functions $m$ for various values of $r$ (c). The calculated $K_{E R}(0)$ of mesostructures with vertically embedded plates as functions of $n$ for various values of $m(\mathrm{~d})$. 
TABLE I. Evaluation of the electrorheological coefficients of layered mesostructures at $\theta=0$. The mesostructures consists of PDMS with various other dielectrics. The enhancements of shear moduli are evaluated for mesostructures under an electric field of $10 \mathrm{MV} / \mathrm{m}$. The vacuum permittivity $\varepsilon_{0}$ is $8.854 \times$ $10^{-12} \mathrm{~F} \mathrm{~m}^{-1}$ and the permittivity of PDMS is $2.65 \varepsilon_{0}$.

\begin{tabular}{lccccccc}
\hline \hline & VHB & Natural rubber & Fluorogel & PVDF & $\mathrm{TiO}_{2}$ & $\mathrm{BaTiO}_{3}$ & \multicolumn{1}{c}{ PZT } \\
\hline$\varepsilon / \varepsilon_{0}$ & 4.54 & 7 & 7.35 & 50 & $86 \sim 173$ & $1250 \sim 10000$ & $500 \sim 6000$ \\
$n$ & 1.71 & 2.64 & 2.77 & 18.87 & $32.45 \sim 65.28$ & $471.70 \sim 3773.58$ & $188.68 \sim 2264.15$ \\
$m$ & 1.31 & 1.62 & 1.66 & 0.5 & 0.5 & 0.5 & 0.5 \\
$K_{E R}(0)$ & 0.19 & 0.78 & 0.88 & 14.99 & $27.18 \sim 56.66$ & $142.91 \sim 164.43$ & $107.38 \sim 162.19$ \\
$\Delta G(\mathrm{kPa})$ & 0.90 & 3.67 & 4.16 & 35.16 & $63.77 \sim 132.93$ & $335.32 \sim 385.80$ & $251.96 \sim 380.55$ \\
\hline \hline
\end{tabular}

Since VHB, natural rubber, and fluorogel are relatively compliant, we assume they shear together with PDMS (i.e., Fig. 2(b)) and set $m=\sqrt{n}$ for the mesostructures. On the other hand, we assume the plates of rigid PVDF, $\mathrm{TiO}_{2}, \mathrm{BaTiO}_{3}$, and PZT rotate in the PDMS matrices (i.e., Fig. 4(a)) when the mesostructure subjected to shear force and set $m=0.5$ and $r=2 \%$. The calculated electrorheological coefficients are listed in Table I. We further evaluate the modulus enhancements of the mesotructures under an electric field of $10 \mathrm{MV} / \mathrm{m}$, which is lower than the breakdown field of PDMS $^{29}$ (Table I). The modulus enhancements of existing ERE with filler particles are on the order of $1 \sim 10 \mathrm{kPa} .^{1,6,7}$ It can be seen that the mesostructures of PDMS-nature rubber or PDMS-fluorogel (Fig. 2(b)) can readily provide similar levels of stiffness enhancements as existing ERE. In addition, the mesostructures created by patterning vertical plates of $\mathrm{BaTiO}_{3}$ and PZT in PDMS (Fig. 4(a)) can potentially give giant tunable modulus on the order of $\sim 300 \mathrm{kPa}$. While dielectric saturation of $\mathrm{BaTiO}_{3}$ and PZT can reduce their permittivity under high electric fields, the proposed mesostructures are still expected to be able to achieve relatively high tunable moduli. Furthermore, while defects in the mesostructures can limit the applied electric field ${ }^{30}$ and possible delamination between the filler plates and elastomer matrix can reduce the electrorheological coefficient, careful design, and fabrication may largely eliminate defects and delamination in mesostructures. For example, multiferroic composites with ceramic plates embedded in polymer matrices have been fabricated with few defects or delamination. ${ }^{31}$

In summary, we demonstrate that anisotropic mesostructures of ERE have significant effects on their tunable stiffness. Deformation of the elastomers changes their mesostructural orientations, which vary their electrostatic potential energies and therefore induce tunable stiffness under applied electric fields. We find that a layered mesostructure arranged in parallel configuration (i.e., Fig. 3(b)) give the highest electrorheological coefficient among all orientations of the layered mesostructure. We further propose to design the mesostructures by vertically embedding rigid plates of high-permittivity dielectrics in polymer matrices. Our theoretical and numerical models show that such mesostructures can potentially provide giant tunable stiffness. It is hoped that the current work will help in the experimental design and fabrication of EREs with extraordinary electrorheological effects.

The work was supported by NSF (CMMI-1253495, CMMI-1200515, and DMR-1121107). C.C. acknowledged the financial support from the Australian National Universality by Dean's Travel Grant Award and Vice Chancellor's Travel Grant.

${ }^{1}$ M. Parthasarathy and D. J. Klingenberg, Mater. Sci. Eng. R-Rep. 17, 57 (1996).

${ }^{2}$ H. See, R. Sakurai, T. Saito, S. Asai, and M. Sumita, J. Electrostat. 50, 303 (2001).

${ }^{3}$ J. D. Carlson and M. R. Jolly, Mechatronics 10, 555 (2000).

${ }^{4}$ Z. Varga, G. Filipcsei, and M. Zrinyi, Polymer 47, 227 (2006).

${ }^{5}$ S. J. A. Koh, X. Zhao, and Z. Suo, Appl. Phys. Lett. 94, 262902 (2009).

${ }^{6}$ N. Tangboriboon, A. Sirivat, R. Kunanuruksapong, and S. Wongkasemjit, Mater. Sci. Eng. C 29, 1913 (2009).

${ }^{7}$ B. Liu, S. A. Boggs, and M. T. Shaw, IEEE Trans. Dielectr. Electr. Insul. 8, 173 (2001).

${ }^{8}$ G. Y. Zhou, Smart Mater. Struct. 12, 139 (2003).

${ }^{9}$ L. Chen, X. L. Gong, and W. H. Li, Smart Mater. Struct. 16, 2645 (2007).

${ }^{10}$ J. Wu, X. Gong, Y. Fan, and H. Xia, Smart Mater. Struct. 19, 105007 (2010).

${ }^{11}$ D. L. Don and J. P. Coulter, J. Intell. Mater. Syst. Struct. 6, 846 (1995).

${ }^{12}$ M. R. Jolly, J. D. Carlson, and B. C. Munoz, Smart Mater. Struct. 5, 607 (1996).

${ }^{13}$ L. C. Davis, J. Appl. Phys. 85, 3348 (1999).

${ }^{14}$ A. Dorfmann and R. W. Ogden, Eur. J. Mech. A-Solids 22, 497 (2003).

${ }^{15}$ S. V. Kankanala and N. Triantafyllidis, J. Mech. Phys. Solids 52, 2869 (2004).

${ }^{16}$ A. Dorfmann and R. W. Ogden, Acta Mech. 174, 167 (2005).

${ }^{17}$ P. P. Castaneda and E. Galipeau, J. Mech. Phys. Solids 59, 194 (2011).

${ }^{18}$ Y. Han, W. Hong, and L. Faidley, Int. J. Appl. Mech. 3, 259 (2011).

${ }^{19}$ S. Rudykh, A. Lewinstein, G. Uner, and G. deBotton, Appl. Phys. Lett. 102, 151905 (2013).

${ }^{20}$ Q. Wang, M. Tahir, L. Zhang, and X. Zhao, Soft Matter 7, 6583 (2011).

${ }^{21}$ Q. Wang, L. Zhang, and X. Zhao, Phys. Rev. Lett. 106, 118301 (2011).

${ }^{22} \mathrm{~S}$. Hong and $\mathrm{X}$. Zhao (unpublished).

${ }^{23}$ K. Bertoldi and M. Gei, J. Mech. Phys. Solids 59, 18 (2011).

${ }^{24}$ Q. Wang, M. Tahir, J. Zang, and X. Zhao, Adv. Mater. 24, 1947 (2012).

${ }^{25}$ X. Zhao, W. Hong, and Z. Suo, Phys. Rev. B 76, 134113 (2007).

${ }^{26}$ C. Keplinger, M. Kaltenbrunner, N. Arnold, and S. Bauer, Proc. Natl. Acad. Sci. U.S.A. 107, 4505 (2010).

${ }^{27}$ D. J. Bergman and D. Stroud, Solid State Phys. 46, 147 (1992).

${ }^{28}$ B. Chu, X. Zhou, K. Ren, B. Neese, M. Lin, Q. Wang, F. Bauer, and Q. M. Zhang, Science 313, 334 (2006).

${ }^{29}$ DowCorning, Data sheet of Sylgard 184, Form No. 06-1009-01. AGP8735, 2007.

${ }^{30} \mathrm{Q}$. Wang, Z. Suo, and X. Zhao, Nature Commun. 3, 1157 (2012).

${ }^{31}$ C.-W. Nan, M. I. Bichurin, S. Dong, D. Viehland, and G. Srinivasan, J. Appl. Phys. 103, 031101 (2008). 